\title{
MOLECULAR GAS AND STAR FORMATION IN INTERACTING AND ISOLATED GALAXIES
}

\author{
J.S. YOUNG \\ Department of Physics and Astronomy \\ Univ. of Massachusetts and FCRAO \\ Amherst, MA 01003 USA
}

\begin{abstract}
The results of the FCRAO Extragalactic CO Survey are used to examine the trends regarding the molecular gas distribution, the star formation efficiency, and the global gas surface densities ( $\mathrm{HI}$ and $\mathrm{H}_{2}$ ) in galaxies as a function of environment. Relative to a sample of isolated Sbc-Scd galaxies, the strongly interacting galaxies have more compact gas distributions, a higher mean value for the global star formation efficiency, and a larger fraction of gas in molecular form. Not only is the molecular gas redistributed during interactions, but evidence is presented for an enhanced conversion of atomic to molecular gas as well.

Among the merger remnants, the star formation efficiency is found to vary by almost two orders of magnitude. Part of this variation is shown to be a function of the merger age, in that the star formation efficiency increases with merger age. Additionally, a new result is presented showing that the strongly interacting galaxies exhibit a completely different trend in the star formation efficiency as a function of galaxy size, when compared with the isolated, paired, and cluster galaxies of similar dimensions.
\end{abstract}

\section{Introduction}

Just 20 years ago, galaxy-galaxy interactions were recognized as capable of significantly influencing the evolution of a galaxy through the triggering of bursts of star formation (Larson \& Tinsley 1978). Since stars form in molecular clouds, a thorough understanding of galaxy-galaxy interactions and the subsequent bursts of star formation requires knowledge of the distribution and abundance of the molecular gas from which stars form in a galaxy. It is the inter-comparison of the gas and star-forming properties of 
interacting galaxies with those of isolated galaxies which can most clearly reveal the power of galaxy-galaxy interactions.

The molecular gas observations which will be used in this paper are taken from the FCRAO Extragalactic CO Survey (Young et al. 1995). These observations provide a unique data base among galaxies for the examination of the global gas content and star formation efficiency within and among galaxies as a function of morphology and environment. Using the 14 meter millimeter telescope of the Five College Radio Astronomy Observatory $\left(\mathrm{HPBW}=45^{\prime \prime}\right)$ we have observed 300 galaxies at 1,412 positions, with detections in 236 galaxies (79\%), and major axis observations in 193 galaxies. The sample of 300 galaxies is not complete in any sense, since the galaxies were chosen to cover a wide range of morphological type, luminosity, and environment. The galaxies in the CO Survey were selected at either optical or IR wavelengths; most are bright spirals, with 260 galaxies ranging from type S0 through Sm. Among galaxies which are either brighter than 11th magnitude in the blue or brighter than $15 \mathrm{Jy}$ at $100 \mu \mathrm{m}$, the CO Survey is $80 \%$ complete.

For over half of the galaxies observed, the $45^{\prime \prime} \mathrm{HPBW}$ of the $14 \mathrm{~m}$ telescope corresponds to a linear resolution of $5 \mathrm{kpc}$ or less. Therefore, the molecular gas surface densities we are measuring correspond to regions within galaxies which are considerably larger than the sizes of individual giant molecular clouds, and therefore represent average surface densities over large regions. Distances to the galaxies in the CO Survey sample were derived assuming a Hubble constant of $50 \mathrm{~km} \mathrm{~s}^{-1} \mathrm{Mpc}^{-1}$. The $\mathrm{H}_{2}$ masses used here were derived from major axis $\mathrm{CO}$ observations, assuming a constant $\mathrm{CO} \rightarrow \mathrm{H}_{2}$ proportionality of $2.8 \times 10^{20} \mathrm{~cm}^{-2}\left[\mathrm{~K}\left(T_{\mathrm{R}}\right) \mathrm{km} \mathrm{s}^{-1}\right]^{-1}$ (Bloemen et al. 1986). The global $\mathrm{H}_{2}$ masses derived for luminous spirals have been shown to be statistically accurate to $\sim 40 \%$ (Devereux \& Young 1990), while the absolute value of the $\mathrm{CO} \rightarrow \mathrm{H}_{2}$ proportionality for the Milky Way has been found to also hold for spirals as diverse as M31 (Sb) and M33 (Scd), by observations which resolve individual molecular clouds (cf. Young \& Scoville 1991, Figure 1).

\section{CO Distribution Results}

Studies examining the molecular gas distributions in spiral galaxies were undertaken in the late 1970's and early 1980's, at a time when it was already known that the atomic gas distributions were similar among galaxies. This is perhaps the reason which led to the expectation that the molecular gas distributions would be similar among galaxies, and to the surprising result that few galaxies were like the Milky Way with its strong central peak, absence of gas between 1 and $4 \mathrm{kpc}$ in radius, and molecular annulus between 5 
and $10 \mathrm{kpc}$ in radius (Burton et al. 1975; Scoville \& Solomon 1975). Among the 193 galaxies whose $\mathrm{CO}$ radial distributions were observed as part of the FCRAO Extragalactic CO Survey (Young et al. 1995), most spiral galaxies exhibit a central CO peak and a smooth decrease as a function of radius. The implied surface density of molecular gas far exceeds that of the atomic gas in the centers of luminous spiral galaxies of all types. Overall, the mass of molecular gas in a galaxy is comparable to the mass of atomic gas, with as much as $10^{10} M_{\odot}$ of molecular gas in the inner disk, and a similar amount of atomic gas distributed over a considerably larger surface area.

Only 10 galaxies in the FCRAO CO Survey were found to show central $\mathrm{CO}$ holes, or CO rings in the radial distribution of molecular gas. The galaxies with $\mathrm{CO}$ rings include types Sab-Sbc, with a peak at type $\mathrm{Sb}$, while the type distribution for the entire CO Survey sample peaks at type Sc. The ring radii among these 10 galaxies range from less than $1 \mathrm{kpc}$ to over $20 \mathrm{kpc}$. It is interesting that 2 of the galaxies with central CO holes were suggested in this meeting to have counter-rotating bulges.

In isolated and non-interacting galaxies, the molecular gas is found to be generally located within the inner half of the optical disk. There is also an indication that the $\mathrm{CO}$ extends over a larger fraction of the optical disk in $\mathrm{Sc}$ galaxies relative to $\mathrm{Sa}$ galaxies, or that the $\mathrm{CO}$ distributions in the Sa galaxies are more compact (Young et al. 1995). This result is interesting since the most compact $\mathrm{CO}$ distributions are found in interacting galaxies (Scoville et al. 1986), and given the possibility that some Sa galaxies may grow their bulges by the accretion of satellite galaxies.

\section{Star Formation Efficiency Results}

One of the most fundamental ways to describe the current epoch star formation in a galaxy is through the comparison of the current star formation rate with the mass of gas available to form stars. This yield of young stars per unit mass of molecular gas is what we call the star formation efficiency (SFE). Recent determinations of the SFE in hundreds of galaxies have shown that similar results are obtained on average, independent of whether one uses star formation rates traced by $\mathrm{H} \alpha$ emission $[\mathrm{L}(\mathrm{H} \alpha)]$ or by the far-IR emission [L(IR)] observed by IRAS (Young et al. 1996). The results described below are based on both of these tracers of the high mass star formation rate.

Studies of the star formation efficiency in spiral galaxies of differing morphology indicate that there is no variation in the mean SFE [based on $L(\mathrm{IR}) / M\left(\mathrm{H}_{2}\right)$ or $\left.L(\mathrm{H} \alpha) / M\left(\mathrm{H}_{2}\right)\right]$ as a function of Hubble type for morphological types Sa through Sc (Rengarajan \& Verma 1986; Devereux \& Young 1991; Young et al. 1996). For spiral galaxies of types $\mathrm{Sa}, \mathrm{Sb}$ and $\mathrm{Sc}$, the 
mean star formation efficiency within each type has a value of $L(\mathrm{IR}) / M\left(\mathrm{H}_{2}\right)$ of $4 L_{\odot} / M_{\odot}$. Thus, even though the galaxies have a diverse morphology and bulge size, the observed constancy of the mean SFE with spiral type indicates a remarkable similarity in the star formation process in the disks of these galaxies.

While similar mean values of the star formation efficiency are found for different morphological types among spiral galaxies, as described above, different mean values of the star formation efficiency are found for galaxies in different environments. Not only is the star formation rate elevated in interacting galaxies compared to isolated galaxies (Larson \& Tinsley 1978; Lonsdale, Persson, and Matthews 1984; Joseph \& Wright 1985), but the star formation efficiency is also elevated in merging systems relative to isolated galaxies (Young et al. 1986; Sanders et al. 1986; Solomon \& Sage 1988; Tinney et al. 1990). For the galaxy samples investigated thus far, the star formation efficiency in strongly interacting galaxies has been found to be elevated by a factor of $\sim 5-20$ relative to isolated galaxies, and this enhanced $\mathrm{SFE}$ is independent of whether one uses star formation rates traced by $\mathrm{H} \alpha$ emission or by the far-IR emission observed by IRAS (Young et al. 1996). Interestingly, studies of the SFE in galaxy pairs show that the SFE is not enhanced in many systems (Solomon \& Sage 1988), and that a very close encounter is needed to produce an observable enhancement in the SFE.

Since the earliest investigations of the star formation efficiency in interacting galaxies, it has been known that both the SFE and the dust temperature are elevated in these galaxies (Young et al. 1986; Solomon \& Sage 1988). This trend is illustrated in Figure 1 , where the ratio $L(\mathrm{IR}) / M\left(\mathrm{H}_{2}\right)$ is plotted versus the ratio of 60 to $100 \mu \mathrm{m} I R A S$ flux densities for a sample of 200 galaxies covering a wide range of environment. These results are consistent with a scenario in which the dust in molecular clouds is heated by young stars, and as the star formation efficiency increases, the resulting increase in the energy density of the radiation field heats the dust to higher temperatures. It is interesting that the ratio of far-infrared luminosity to atomic gas mass does not show a tight correlation like that in Figure 1, supporting the suggestion that it is primarily the dust in molecular clouds which is radiating at the $30-40 \mathrm{~K}$ dust temperatures indicated by the IRAS data.

Among the merging and closely interacting galaxies, a wide spread in the SFE is observed, a spread which is wider than the factor of 10 observed spread in SFE for the isolated galaxies. This scatter is also considerably larger than the measurement uncertainties, and is probably related to the age of a merger (Young et al. 1986). Joseph \& Wright (1985) have suggested that the age of a merger can be estimated qualitatively based on the presence of two distinct disks in the younger systems (such as NGC 


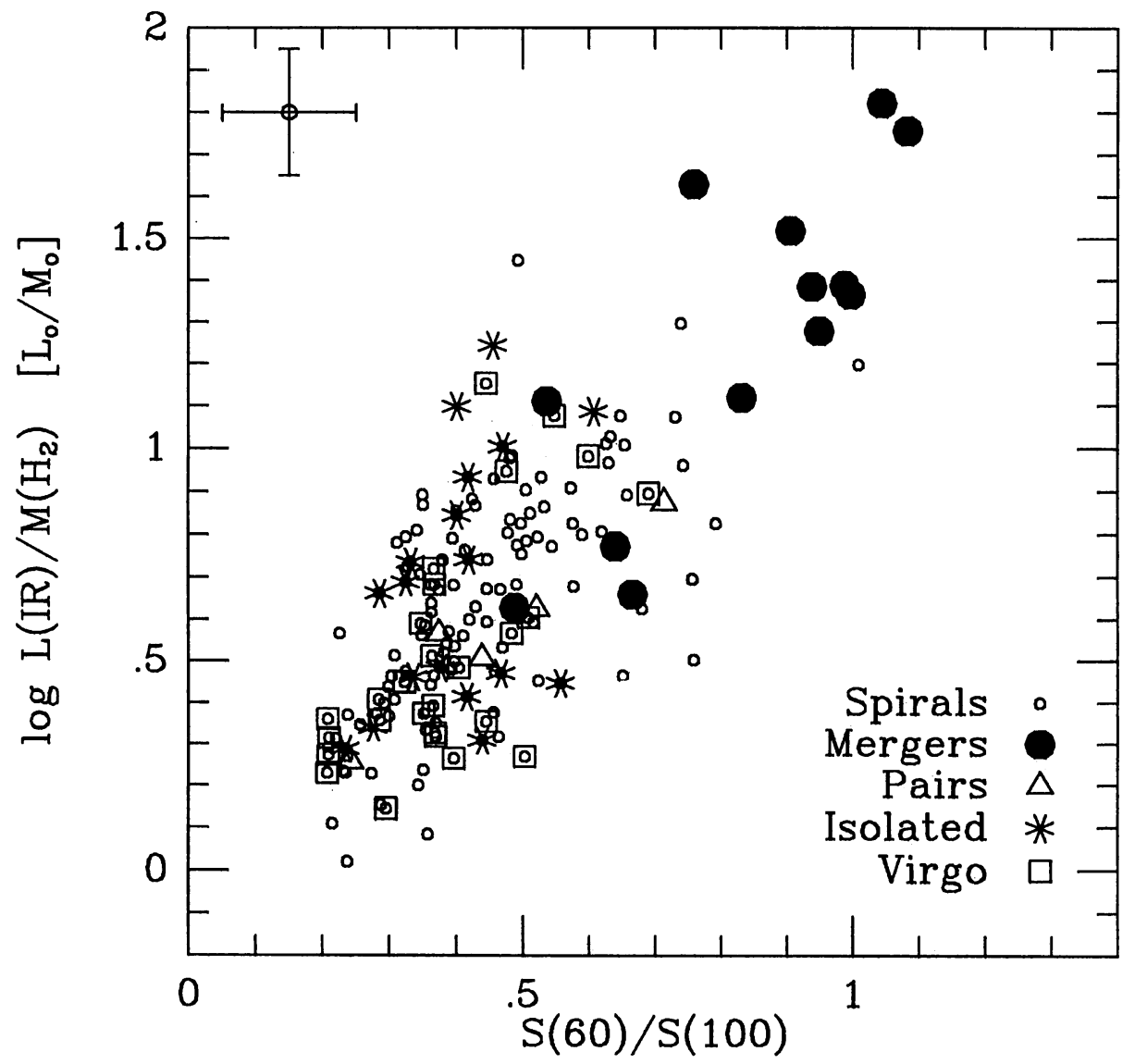

Figure 1. Comparison of the ratio $L(\mathrm{IR}) / M\left(\mathrm{H}_{2}\right)$ with the ratio of $I R A S 60 / 100 \mu \mathrm{m}$ flux densities for 200 spiral galaxies in the FCRAO Extragalactic CO Survey (Young et al. 1995). The galaxies are coded by environment as indicated. Note that the merging/interacting galaxies have the highest values of both the SFE and $S(60) / S(100)$ relative to the isolated galaxies, although the intermediate SFE values are shared by galaxies in all environments. Part of the scatter in the SFE for the merging and interacting galaxies results from an increase in the SFE with merger age (see text).

4038/39 and NGC 520), and that as a merger ages, the nuclei are found closer together, until the merger remnant resembles a single galaxy (such as NGC 3310). Since many of the galaxies in our sample are also in the sample of Joseph \& Wright (1985), we have used their estimates of merger age in relation to the values of $L(\mathrm{IR}) / M\left(\mathrm{H}_{2}\right)$. We find that the lowest star formation efficiencies are found in the youngest systems, and the highest values in the oldest systems, indicating that the star formation efficiency increases with merger age (Young et al. 1986). 
A new result regarding the star formation efficiency in interacting galaxies was uncovered in preparing the talk for IAU 186. It is clear from Figure 1 that there is a considerable spread in the star formation efficiency among galaxies found in similar environments. Some of this scatter in the SFE for closely interacting galaxies is related to the merger age, as described above. An additional source of scatter in the star formation efficiency among galaxies in similar environments is shown in Figure 2 to be galaxy size.

In Figure 2, the ratio $L(\mathrm{IR}) / M\left(\mathrm{H}_{2}\right)$ is plotted versus the galaxy optical linear diameter $[D(25)]$ for galaxies in different environments. Among the isolated galaxies, Virgo galaxies, pairs, and field spirals, there is a trend of decreasing mean star formation efficiency with increasing galaxy size. For 18 galaxies smaller than $16 \mathrm{kpc}$ in diameter, the mean SFE is $9 \pm 1$ $L_{\odot} / M_{\odot}$, while for 25 galaxies larger than $50 \mathrm{kpc}$ in diameter, the mean $\mathrm{SFE}$ is $3 \pm 0.5 L_{\odot} / M_{\odot}$. In sharp contrast to this trend in the SFE with galaxy size, the merging and closely interacting galaxies behave completely differently. At every galaxy size, the mergers have the highest star formation efficiencies for galaxies of that size, and there seems to be an upper limit to the SFE independent of galaxy size. The mergers with the lowest SFE values are found to be among galaxies of the largest size, and these are the young mergers referred to above. Even with these low SFE values, the star formation efficiencies in the large merging systems are as high as the highest values found in non-merging systems of the same size.

The reason for the observed trend of decreasing star formation efficiency with increasing galaxy size is not entirely clear. This effect could possibly be related to the triggering of star formation in normal galaxies. A triggering event such as an individual supernova explosion will impact on a much larger fraction of the area of a small galaxy than a similar event in a much larger galaxy. Additionally, there is a significant difference in the dynamics of a large galaxy versus a small galaxy. A smaller galaxy tends to have a rotation curve which barely turns over, leading to almost solid body rotation over most of the galaxy, while a larger galaxy with a flat rotation curve over almost the entire disk will experience shear throughout (Rubin et al. 1985). The effect of this shear could be to spread out the effects of a star formation trigger, and lessen its efficiency. Thus, given two galaxies with the same mass of gas, the above conditions could lead to higher star formation rates (and therefore higher efficiencies) in the smaller galaxies. Furthermore, the effect of a galaxy-galaxy encounter is to enhance the triggering of star formation on the galaxy wide scale, so that the large merging galaxies develop star formation efficiencies which exceed those of most non-interacting galaxies of the same size. 


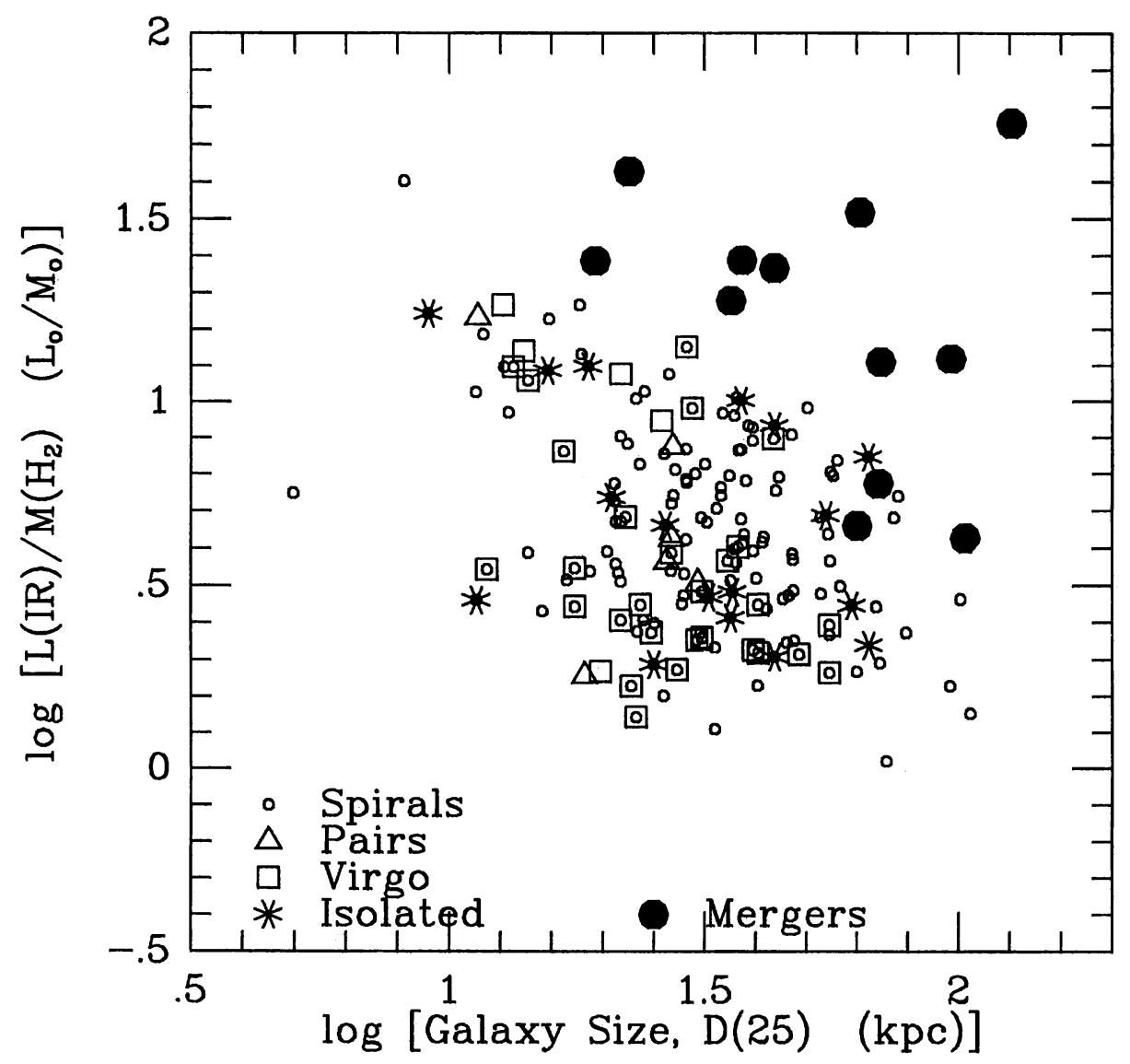

Figure 2. Comparison of the ratio $L(\mathrm{IR}) / M\left(\mathrm{H}_{2}\right)$ with the linear diameters $[D(25)]$ of galaxies for 200 spiral galaxies in the FCRAO Extragalactic CO Survey. The galaxies are coded by environment as indicated. Among isolated, paired and cluster galaxies, there is a trend of decreasing SFE with increasing galaxy size. The merging/interacting galaxies exhibit a completely different behavior in the SFE with galaxy size.

\section{Molecular and Atomic Gas Compared}

Since star formation occurs in molecular clouds, and molecular clouds form from atomic gas, the relative amounts of atomic and molecular gas will both have an influence on the future star formation in a galaxy. For this reason, it is important to examine the global $\mathrm{H}_{2} / \mathrm{HI}$ ratio and the globally averaged molecular and atomic gas surface densities in order to constrain models which describe the gas and star formation in galaxies. Furthermore, it is necessary to examine galaxies in a range of physical environments in order to determine the prevailing conditions surrounding the most extreme 
variations in the atomic and molecular gas content.

Among non-interacting spiral galaxies, the global ratio of molecular to atomic gas mass shows a decreasing trend along the Hubble sequence (Young \& Knezek 1989; Thronson et al. 1989), with more molecular than atomic gas in early type spirals ( $\mathrm{S} 0$ and $\mathrm{Sa}$ ) and more atomic than molecular gas in late type spirals (Sc). In determining the mean ratio of $\mathrm{H}_{2} / \mathrm{HI}$ for galaxies of a specific morphological type, it is important to exclude galaxies which are in disturbed environments or in clusters, where the atomic gas may have been stripped from the disk. To this end, we have recently made a careful analysis of the $\mathrm{H}_{2} / \mathrm{HI}$ ratio in galaxies for the entire FCRAO Extragalactic CO Survey. Within this sample, we have sufficient statistics to examine the ratio of molecular to atomic gas in isolated galaxies, in closely interacting and merging galaxies, in Virgo cluster spirals, and in non-Virgo spirals along the Hubble sequence.

Among isolated galaxies and non-interacting field galaxies, we confirm the trend of a decreasing mean value in the molecular to atomic gas ratio along the Hubble sequence, with $\mathrm{H}_{2} / \mathrm{HI}=1.6$ in the Sa's and 0.4 in the Sc's. It is interesting that spiral galaxies of types Sa through Sc have similar mean molecular gas surface densities, so that the changing global ratio of molecular to atomic gas results primarily from variations in the HI content of spiral galaxies with type. Thus, the mean gas surface densities $\left(\mathrm{HI}+\mathrm{H}_{2}\right)$ increase a factor of 2 from type Sa to Sc.

With regard to the effects of environment on the gas properties in galaxies, the molecular and atomic gas surface densities and $\mathrm{H}_{2} / \mathrm{HI}$ ratio in the isolated galaxies (types Sbc-Scd) resemble those of the Sc galaxies. It is the mergers and close pairs which exhibit the most unusual gas properties among galaxies. The mean total gas surface density for the pairs and mergers is, not surprisingly, like that for 2 spirals (i.e. 2 Sc galaxies or an $\mathrm{Sc}$ and an $\mathrm{Sa}$, but not 2 Sa galaxies). While the mean atomic gas surface density among the pairs and mergers is similar to that for an Sc or an isolated galaxy, the mean molecular gas surface density is a factor of 3 higher (cf. Braine \& Combes 1993). Therefore, the mean $\mathrm{H}_{2} / \mathrm{HI}$ ratio in pairs and mergers is 2, four times higher than that in the isolated (Sbc-Scd) galaxies and slightly higher than that in the Sa galaxies. This probably reflects the enhanced conversion of atomic to molecular gas in interactions as well as the removal of some of the more extended HI (Scoville et al. 1986; Mirabel \& Sanders 1989). Thus, the dominant gas phase changes from $\mathrm{HI}$ in isolated Sbc-Scd galaxies to $\mathrm{H}_{2}$ in interactions.

It has always seemed curious to me that only the galaxy mergers, and not the galaxy pairs, exhibit an enhanced star formation efficiency, since clearly many galaxy pairs (NGC 4038/39, for example) show enhanced rates of star formation. The above analysis indicates that the galaxy pairs 
do show elevated molecular gas surface densities. Therefore, the reason why no enhancement is found in the star formation efficiency is that the infrared surface brightnesses in the galaxy pairs are also elevated by the same amount.

\section{Discussion and Conclusions}

The results described here, with regard to the trends in the star formation efficiency and the molecular to atomic gas ratio as a function of morphology and environment, provide constraints for theories of the formation and evolution of galaxies, especially with regard to the Hubble sequence and to the effects of galaxy-galaxy interactions.

In spiral galaxies of types Sa-Sc, the mean star formation efficiency does not vary with Hubble type, while the ratio of molecular to atomic gas decreases by a factor of 4 among the same systems. These results can be understood in terms of the size scale on which gravity plays a role in cloud and star formation. To form the molecular clouds, atomic gas on a large scale must be assembled, so that perhaps it is not surprising that the $\mathrm{H}_{2} / \mathrm{HI}$ ratio changes with galaxy morphology, or equivalently, with the mass distribution in a galaxy. Once molecular clouds are formed, however, the constant star formation efficiency with galaxy type indicates that star formation is primarily a local process, with gravity on the small scale playing the most important role. Only when a large scale perturbation is present, such as in a bar, a spiral arm, or a tidal encounter with another galaxy, does the star formation efficiency become elevated. This enhanced SFE generally reflects an enhanced concentration of molecular gas.

In merging and interacting galaxies, the gas and star formation are strongly influenced by the interaction, with enhancements in both the global ratio of molecular to atomic gas and the star formation efficiency in merging systems. Not only is the molecular gas redistributed and the atomic gas possibly stripped during an interaction, but enhanced conversion of atomic to molecular gas in interacting galaxies also seems to be required. The triggering of star formation on a galaxy-wide scale then elevates the star formation efficiency by a factor of $\sim 5-20$ over that observed in isolated galaxies. As a merger ages, we find that the SFE increases, probably reflecting the cumulative effects of both an increase in the luminosity from ongoing star formation, and the consumption of the molecular gas to form stars.

The future of star formation in the currently merging systems is not clear. This is in part because of the inability to measure the low mass end of the initial mass function. For the "extended" Miller-Scalo IMF (Kennicutt 1983), and given the present rates of high mass star formation and the 
global masses of interstellar gas in the galaxies we have studied, the gas will be cycled through stars in $10^{8}$ to $10^{10}$ years. However, some galaxies (i.e. M82 and NGC 2146) will cycle the gas in the center in $10^{9}$ years, while the gas in the outer parts can sustain the present rate of star formation for $10^{11}$ years. Thus, gas depletion may not occur everywhere, and not at all if only high mass stars form.

\section{References}

Burton, W.B., Gordon, M., Bania, T., \& Lockman, F.J. (1975), Ap.J., 202, 30.

Devereux, N., \& Young, J.S. (1990), Ap.J., 359, 42.

Devereux, N., \& Young, J.S. (1991), Ap.J., 371, 515.

Joseph, R.D., \& Wright, G.S. (1985), M.N.R.A.S., 214, 87.

Kennicutt, R.C. (1983), Ap.J., 272, 54.

Larson, R., \& Tinsley, B. (1978), Ap.J., 219, 46.

Lonsdale, C., Persson, E., \& Matthews, K. (1984), Ap.J., 287, 95.

Mirabel, F., \& Sanders, D.B. (1989), Ap.J.(Letters), 340, L53.

Rengarajan, T.N., \& Verma, R.P. (1986), Astr.Ap., 165, 300.

Rubin, V.C., Burstein, D., Ford, W.K., \& Thonnard, N. (1985), Ap.J., 289, 81.

Sanders, D.B., et al. (1986), Ap.J.(Letters), 305, L45.

Scoville, N., \& Solomon, P.M. (1975), Ap.J.(Letters), 199, L105.

Scoville, N., et al. (1986), Ap.J.(Letters), 311, L47.

Solomon, P.M., \& Sage, L. (1988), Ap.J., 334, 613.

Thronson, H., et al. (1989), Ap.J., 344, 747.

Tinney, C., et al. (1990), Ap.J., 362, 473.

Young, J.S., Kenney, J., Tacconi, L., Claussen, M., et al. (1986), Ap.J., 311, L17.

Young, J.S., Xie, S., Tacconi, L., Knezek, P., et al. (1995), Ap.J.Suppl., 98, 219.

Young, J.S., Allen, L., Kenney, J., Lesser, A., \& Rownd, B. (1996), A.J., 112, 1903.

Young, J.S., \& Knezek, P. (1989), Ap.J.(Letters), 347, L55.

Young, J.S., \& Scoville, N.Z. (1991), Ann.Rev.Astr.Ap., 29, 581. 